\title{
PHENOTYPIC ASSAYS FOR DETECTION OF EXTENDED SPECTRUM B- LACTAMASES AND CARBAPENEMASES: A LABORATORY GUIDE FOR MICROBIOLOGISTS
}

\author{
Dickson Aruhomukama ${ }^{1^{*}}$ \\ 1Department of Medical Microbiology, College of Health Sciences, Makerere University, \\ P.O Box 7072, Kampala, Uganda.
}

*Correspondence: Dickson Aruhomukama; dickson.aruhomukama@chs.mak.ac.ug

\section{Abstract}

This review aimed at defining and classifying extended spectrum $\beta$-lactamases, ESBLs and carbapenemases, summarizing the different phenotypic methods used to detect production of these enzymes in clinically significant gram negative bacteria and also describing the methods that discourse challenges mostly encountered during detection of these enzymes in microbiology laboratories with the purpose of formulating recommendations on best practice to screen for these enzymes. We conclude that the modified double disk synergy, MDDS is not only suitable for the confirmation of ESBL production after screening isolates with the cephalosporin/clavulanate combination disc diffusion or broth micro-dilution methods but also distinguishes ESBL production and over-expression of AmpC-derepressed mutants and as well serves as an indicator for AmpC screening. Furthermore, we suggest cefotaxime, ceftazidime and cefpodoxime (for testing using a single drug) as indicator antibiotics of choice for ESBL detection. The MDDS and cefoxitin/AmpC inhibitor combination disc method, using cloxacillin and phenylboronic acid can be used as screening tests for AmpC production and either the AmpC disc test, the disc approximation test or the modified three dimensional extract test as confirmatory tests for AmpC production. We also suggest that confirmation of carbapenemase production be done with the modified hodge test, using Klebsiella pneumoniae ATCC 700603 as the indicator organism or the modified carbapenem inactivation method. However, to differentiate between the different classes of carbapenemases, boronic acid and EDTA based methods (double-disk synergy tests and 
combined-disk tests) using imipenem, meropenem and ertapenem, in combination with 3-aminophenylboronic acid and ethylene diamine tetra acetic acid be used.

Keywords: Extended spectrum $\beta$-lactamases, AmpC Carbapenemases, Carbapenemases, Classification, Phenotypic Detection, Clinically significant bacteria

\section{Introduction}

Infections involving gram negative Extended Spectrum $\beta$-lactamase and Carbapenemase producing bacteria in Health-Care settings continue to increase [1-6]. The treatment of these infections remains a serious public health concern, as even the most likely nonfatal infections become fatal when they involve particularly these antibiotic resistant bacteria [1, 2, 5-8]. Extended Spectrum $\beta$-lactamase, ESBL and Carbapenemase enzymes hydrolyze and hence inactivate $\beta$-lactam antibiotics resulting in $\beta$-lactam resistance in bacteria that are producers of these enzymes [6, 9-13]. Increased $\beta$-lactam resistance in bacteria has been attributed to their continuous exposure to $\beta$-lactam antibiotics, a factor that has contributed to increased uninterrupted production and mutation of $\beta$-lactamases in bacteria $[6,14]$. Despite the Clinical Laboratory Standards Institute, CLSI recommendations in regards detection of ESBL and Carbapenemase production in clinically significant bacteria, the occurrence of ESBL and Carbapenemase producing bacteria remains extremely difficult to resolve, this is attributed to various reasons notably; i) difficulty in detecting ESBL and Carbapenemase production in these bacteria; ii) inconsistences in the reporting of results in regards ESBL and Carbapenemase production and iii) co-existence of $\beta$-lactamase enzymes in the bacteria $[6,7,15,16]$. These therefore set precedence for more accurate and practical phenotypic approaches to the detection of ESBLs and Carbapenemases in bacteria, as these are beneficial in providing substantial information in regards ESBLs and Carbapenemases in these bacteria. Additionally, these phenotypic approaches are also vital in reducing the need to apply the more expensive molecular techniques for detection of production of these enzymes in bacteria. Hence, this review defines and classifies ESBLs and Carbapenemases and summarizes the different phenotypic methods used in the 
detection of ESBLs and Carbapenemases in bacteria. Noteworthy, also described herein are methods that discourse challenges in detection of ESBLs and Carbapenemases.

\section{Extended Spectrum $\beta$-lactamases: Definition and Classification}

Extended Spectrum $\beta$-lactamases, ESBLs are enzymes that break down and thus inactivate antibiotics known as $\beta$-lactams $[6,12,13,17]$. Additionally, ESBLs are characterized by their susceptibility to inhibition by $\beta$-lactam inhibitors particularly; clavulanic acid, tazobactam and sulbactam [6,12, 13]. $\beta$-lactamases are classified according to two schemes, these are; i) the Bush-Jacoby-Medeiros functional classification and ii) the Ambler molecular classification $[6,12,13]$. The basis of the BushJacoby-Medeiros functional classification is the functional properties of the $\beta$-lactamase enzymes principally, their inhibitor and substrate profiles [12, 13]. The Ambler molecular classification however is based on the protein homology of the $\beta$-lactamase enzymes $[12$, 13]. The Ambler molecular classification further classifies $\beta$-lactamase enzymes into four classes namely; class $A, C$ and $D$ also known as the serine $\beta$-lactamases and class $B$ lactamases also known as the zinc or Metallo $\beta$-lactamases $[12,13]$.

The CTX-M type forms the most common genetic variant of ESBLs, this family of $\beta$ lactamases specially hydrolyze cefotaxime over ceftazidime and are found exclusively in the functional group $2[6,12,18-21]$. Also, unique to these $\beta$-lactamases is their susceptibility to inhibition by the $\beta$-lactam inhibitor tazobactam as compared to inhibition by the other $\beta$-lactam inhibitors, clavulanic acid and sulbactam [6, 20, 22]. The Extended Spectrum of activity of the CTX-M $\beta$-lactamases is attributed to the serine residue present at position 237 [6, 21]. The CTX-M $\beta$-lactamases can be divided into five groups namely; the CTX-M group 1, 2, 8, 9 and 25 basing on their amino acid sequences [6, 12, 23]. unlike the other $\beta$-lactamase enzymes, the CTX-M enzymes are acquired and disseminated in bacteria via mobile genetic elements particularly conjugative plasmids and transposons in horizontal gene transfer processes [6].

The TEM type $\beta$-lactamases form another $\beta$-lactamase family; $\beta$-lactamases belonging to the TEM type, TEM-1 a variant of TEM hydrolyze penicillins and first generation cephalosporins [6]. The TEM-3 $\beta$-lactamases, which are another variant of TEM $\beta$ lactamases hydrolyze Extended Spectrum Cephalosporins [6, 24, 25]. In addition to these 
families is the SHV type $\beta$-lactamases, SHV-1 $\beta$-lactamases, a variant of the SHV hydrolyze broad spectrum penicillins, these include ampicillin, tigecycline and piperacillin $[25,26]$; the OXA type $\beta$-lactamases, these $\beta$-lactamases exhibit the ability to hydrolyze oxacillin, are predominantly present in Pseudomonas aeruginosa and Acinetobacter baumannii however also occur in other gram negative bacteria [6, 12, 27, 28]; the PER type $\beta$-lactamases, unique about these $\beta$-lactamases is their efficient hydrolysis of penicillins, cephalosporins and their susceptibility to clavulanic acid inhibition $[6,12,13]$; the GES type $\beta$-lactamases, these $\beta$-lactamases hydrolyze penicillins and Extended Spectrum Cephalosporins, but not cephamycins or carbapenems, these are also inhibited by $\beta$-lactamase inhibitors $[6,12,13]$; other described $\beta$-lactamase families include among others the VEB-1, BES-1, CME-1 and SFO-1, enzymes belonging to these families have mostly been found to exhibit $\beta$-lactamase activity $[6,29,30]$.

\section{Carbapenemases: Definition and Classification}

Carbapenemases are enzymes that break down and thus inactivate antibiotics known as carbapenems, these enzymes represent the most versatile family of $\beta$-lactamases and are uniquely characterized by their broad spectrum of activity as compared to other $\beta$ lactam hydrolyzing enzymes [12, 13, 31-35]. Carbapenemases are classified into two major groups basing on the nature of their active sites, these groups are the serine carbapenemases belonging to the class $A$ penicillinases and class $D$ oxacillinases and the Metallo $\beta$-lactamases belonging to the class $B$ carbapenemases which contain one or more zinc atoms at their active sites a characteristic future that allows them to hydrolyze the bicyclic $\beta$-lactam ring $[12,13,31-35]$.

Class A Carbapenemases, also known as the class A serine carbapenemases, when present in bacteria confer a characteristic reduced susceptibility to imipenem [31, 35]. Class A carbapenemases include; IMI/NMC, KPC, GES and SME enzymes [31, 35]. These enzymes require an active serine at position 70 for their hydrolytic activity in the Ambler numbering system for class A $\beta$-lactamases, hydrolyze carbapenems, cephalosporins, penicillins and aztreonam, are inhibited by clavulanic acid and tazobactam [31, 35, 36]. IMI, NMC and SME are chromosomally encoded enzymes whereas GES and KPC enzymes are plasmid encoded [31, 35]. 
Class B carbapenemases, also known as class B Metallo $\beta$-lactamases hydrolytic activity is dependent on the interaction of the $\beta$-lactams with zinc ions in the active sites of these enzymes, these interactions result in the distinctive trait of their inhibition by EDTA which is a chelator of zinc and other divalent ions [31, 33, 35, 37, 38]. These enzymes ably hydrolyze carbapenems, are resistant to commercially available $\beta$-lactamase inhibitors, are susceptible to inhibition by metal ion chelators and have a relatively broad substrate profile that includes cephalosporins and penicillins [31, 33, 35, 37, 38]. Also, characteristic to the class $B$ carbapenemases is their inability to hydrolyze aztreonam $[31,33,35,37$, 38]. These enzymes include; GIM, IMP, VIM, NDM and SIM [31, 35, 38]. Additionally, these enzymes mostly occur in integron structures and are mostly disseminated via mobile genetic elements particularly conjugative plasmids and transposons in horizontal gene transfer [31, 35, 38, 39].

Class $D$ carbapenemases, also known as class $D$ Metallo $\beta$-lactamases or the OXA $\beta$ lactamases are uniquely characterized by their association with plasmids, hence are mostly plasmid encoded, although may also be chromosomally encoded [31, 35, 40-42]. Additionally, these enzymes hydrolyze oxacillin and cloxacillin and are described as penicillinases, are poorly inhibited by clavulanic acid and EDTA and have large amounts of variability in their amino acid sequences [12, 31, 35, 42, 43].

\section{Detection of Extended Spectrum $\beta$-lactamase Production}

Different phenotypic methods, disk diffusion-based methods and broth micro-dilution methods have been described previously to ably detect the production of ESBLs in clinically significant bacteria [7]. However, currently some of these methods have become increasingly unreliable, this is attributed to; i) the co-existence of different classes of $\beta$ lactamases in bacteria [44-46], ii) difficulty in recognition of ESBL production due to the over-expression of AmpC $\beta$-lactamases [44-46], iii) potential masking of ESBL production by AmpC producing bacteria which serve as reservoirs of ESBLs [44-46], and also iv) the general inability of most microbiology laboratories to ably perform testing for the detection of ESBL production in bacteria [47-50], all of which affect testing and consequently result in inappropriate antimicrobial therapy $[44,51]$. In the testing for ESBL production, other 
factors including inoculum density [52-54] and the distance between the discs [7, 53-56] being tested have been documented to influence the outcomes of the testing.

Testing for ESBL production in bacteria is a two stage process that involves; screening and confirmation of production of ESBLs in potential producing bacterial isolates [57, 58]. Screening and confirmation of ESBL production follows testing bacteria against array of antibiotics namely; cefpodoxime, ceftazidime, ceftriaxone, cefotaxime and aztreonam and identifying specific zone diameters indicative of potential ESBL production [57, 58]. Testing with greater than one of the antibiotics is recommended to improve the sensitivity of ESBL detection $[57,58]$. It is however recommended to use cefotaxime, due to its consistent susceptibility to CTX-M and ceftazidime, due its consistent susceptibility to TEM and SHV [57, 59-61]. Cefpodoxime however should be used when testing is to be performed using a single drug [57, 59-61]. Reduced susceptibility to any of the agents as specified by the Clinical Laboratory Standards Institute, CLSI is a qualification of ESBL testing and necessitates phenotypic confirmatory testing to confirm diagnosis [58]. Phenotypic confirmation of ESBL production can be achieved using array of methods, including the use of the cephalosporin/clavulanate combination disc diffusion or broth micro-dilution method [57, 58], the double disk synergy (DDS) method [6, 56, 57, 62-64] and a modification of this method, the modified double disc synergy (MDDS) method, which confirms ESBL production and also differentiates ESBL production and overexpression of AmpC-derepressed mutants [6, 56, 57, 62-64].

In the cephalosporin/clavulanate combination disc diffusion and broth micro-dilution methods; testing is performed following the CLSI guidelines for susceptibility testing using both cefotaxime $(30 \mu \mathrm{g} / 0.25-64 \mu \mathrm{g} / \mathrm{ml})$ and ceftazidime $(30 \mu \mathrm{g} / 0.25-128 \mu \mathrm{g} / \mathrm{ml})$ alone or in combination with clavulanic acid (10 $\mu \mathrm{g}$ or $0.25 / 4-64 / 4 \mu \mathrm{g} / \mathrm{ml} / 0.25 / 4-128 / 4 \mu \mathrm{g} / \mathrm{ml}$ ) [58]. Reporting of results is done after $16-20$ hours of incubation in ambient air at $35^{\circ} \mathrm{C} \pm 2^{\circ} \mathrm{C}$ as follows; in the disc diffusion method, an increase by $\geq 5 \mathrm{~mm}$ in the zone of inhibition from either antimicrobial agent tested in combination with the clavulanate versus the zone diameter of the agent when tested alone is indicative of ESBL production [58]. In the broth micro-dilution, $a \geq 3$ two fold concentration decrease in the Minimum Inhibitory 
Concentration, MIC for either antimicrobial agent tested in combination with clavulanate versus the MIC of the agent when tested alone is indicative of ESBL production [58].

In the DDS, testing is performed following the CLSI guidelines for susceptibility testing using cefotaxime $(30 \mu \mathrm{g})$ and/or ceftriaxone $(30 \mu \mathrm{g})$ and/or ceftazidime $(30 \mu \mathrm{g})$ and/or aztreonam $(30 \mu \mathrm{g})$ and a disc of amoxicillin-clavulanic acid $(20 / 10 \mu \mathrm{g})$ at a distance of $30 \mathrm{~mm}$ center to center; However, narrower distances between the discs have been associated with increased sensitivity of the test and have been previously described [7, $56,65-67]$. Reporting of the results is done after incubation in ambient air at $35^{\circ} \mathrm{C} \pm 2{ }^{\circ} \mathrm{C}$ for 16-20 hours as follows; a decreased susceptibility to the antibiotic disc used combined with a clear-cut enhancement of the inhibition zone of same antibiotic disc in front of the clavulanic acid containing disc, often resulting in a characteristic shape-zone referred to as "champagne-cork" or key hole is indicative of ESBL production whereas absence of the same is indicative of non ESBL production [7, 56, 65-67].

In the MDDS, the following antibiotic discs are used; amoxicillin-clavulanic acid (20/10 $\mu \mathrm{g})$ or piperacillin tazobactam $(100 / 10 \mu \mathrm{g})$ along two third generation cephalosporins, ceftazidime $(30 \mu \mathrm{g})$ and cefotaxime $(30 \mu \mathrm{g})$; a fourth generation cephalosporin, cefepime $(30 \mu \mathrm{g})$ used as a replacement for ceftriaxone $(30 \mu \mathrm{g})$ and aztreonam $(30 \mu \mathrm{g})$ [7, 63-65]. This test is performed following the CLSI guidelines for susceptibility testing as follows; cefotaxime $(30 \mu \mathrm{g})$ is placed $20 \mathrm{~mm}$ from the amoxicillin-clavulanic acid $(20 / 10 \mu \mathrm{g})$, aztreonam $(30 \mu \mathrm{g})$ disc is placed at $25 \mathrm{~mm}$, ceftazidime $(30 \mu \mathrm{g})$ at $30 \mathrm{~mm}$ and cefepime $(30 \mu \mathrm{g})$ at $30 \mathrm{~mm}$, incorporation of this modification into a gram negative template involves placing a piperacillin tazobactam $(100 / 10 \mu \mathrm{g})$ disc $25 \mathrm{~mm}$ from cefepime $(30 \mu \mathrm{g})[7,63-$ 65]. Reporting of results is done after $16-20$ hours of incubation in ambient air at $35^{\circ} \mathrm{C} \pm$ $2^{\circ} \mathrm{C}$ as follows; Strains are divided into three groups basing on interpretation with MDDS and their susceptibilities to cefotaxime and ceftazidime, Group 1 (Wild type) includes ESBL non producers sensitive to cefotaxime and ceftazidime; Group 2 (derepressed mutants) includes ESBL non producers resistant to cefotaxime and ceftazidime; Group 3 (ESBL producers) includes ESBL producers sensitive, intermediate or resistant to cefotaxime and ceftazidime [7, 63-65]. Worth mentioning, reporting of results is done 
following CLSI guidelines as follows; isolates with a positive confirmatory test are reported as resistant to all penicillins, cephalosporins and aztreonam except for cephamycins and cefoxitin irrespective of the MIC of the particular cephalosporin [7, 63-65].

\section{Detection of AmpC $\beta$-lactamase production}

AmpC $\beta$-lactamases are enzymes that belong to class $C$ in the Ambler structural classification or group 1 of the Bush's functional classification [13, 46, 68, 69]. AmpC $\beta$ lactamases may be chromosomally encoded, chromosomal AmpCs [69-71] or plasmid mediated, plasmid-mediated AmpCs [68, 69, 71, 72]. Both types of AmpC $\beta$-lactamases have been studied and documented to occur in clinically significant bacteria $[45,68,71$, 73]. These enzymes confer resistance to $\beta$-lactam antibiotics notably penicillins, third generation cephalosporins, cephamycins (cefoxitin and cefotetan) and monobactams notably aztreonam $[45,69,71,74]$. Bacterial producers of these enzymes are however susceptible to fourth generation cephalosporins (cefepime and cefpirome) [46, 69], are also poorly inhibited by clavulanic acid $[46,69]$, hence are resistant to $\beta$-lactam/ $\beta$ lactamase-inhibitor combinations and give a positive and negative test during ESBL screening and confirmation respectively [68]. These features form the basis of screening and confirmation of AmpC $\beta$-lactamase production in bacteria $[68,69,75,76]$.

Screening for the production of AmpC $\beta$-lactamases in bacteria is done using cefoxitin $(30 \mu \mathrm{g})$ and cefoxitin $(30 \mu \mathrm{g})$ supplemented with an AmpC inhibitor either 200 $\mathrm{\mu g}$ of cloxacillin or $400 \mu \mathrm{g}$ of phenylboronic acid or using cefpodoxime $(10 \mu \mathrm{g})$ and cefpodoxime $(10 \mu \mathrm{g})$ supplemented with an AmpC inhibitor, either $200 \mu \mathrm{g}$ of Cloxacillin or $400 \mu \mathrm{g}$ of Phenylboronic acid [76-80]. Reporting of results is done after 16-20 hours of incubation in ambient air at $35^{\circ} \mathrm{C} \pm 2^{\circ} \mathrm{C}$ as follows; an increase in the zone of inhibition by $\geq 4 \mathrm{~mm}$ in the cefoxitin or cefpodoxime discs supplemented with either of the AmpC inhibitors as compared to the unsupplemented disc of either cefoxitin or cefpodoxime is indicative of AmpC production [76-79, 81].

Another similar method with minor modifications that can be used to screen for the production of AmpC $\beta$-lactamases in bacteria [65] is performed by making a lawn culture of E.coli ATCC 25922 adjusted to a 0.5 McFarland standard on Mueller Hinton agar, MHA 
following the CLSI guidelines for standard disc diffusion testing [58, 65]. Following this, a cefoxitin $(30 \mu \mathrm{g})$ disc is placed on the surface of the inoculated MHA plate [65]. Then a sterile plain $6 \mathrm{~mm}$ paper disc initially inoculated with several colonies of the test organism is placed besides the cefoxitin disc almost making contact with it [65]. Reporting of results is done after $16-20$ hours of incubation in ambient air at $35^{\circ} \mathrm{C} \pm 2^{\circ} \mathrm{C}$ as follows; indentation or a flattening of the inhibition zone is indicative of enzyme inactivation of the cefoxitin hence $\mathrm{AmpC}$ production whereas the absence of distortion is indicative of non-significant enzyme inactivation of the cefoxitin hence non AmpC production [65].

Phenotypic confirmation of $\mathrm{AmpC}$ production is done by performing either the following tests; i) the AmpC disc test [45, 68], ii) the disc approximation test $[64,78,80,81]$ or iii) the modified three dimensional extract test [64, 80, 82-84].

In the AmpC disc test, a plate of MHA is inoculated with a lawn culture of E.coli ATCC $25922[45,68]$. Following this, sterile $6 \mathrm{~mm}$ paper discs infused with $20 \mu \mathrm{l}$ of $1: 1$ Tris-EDTA : saline solution or not infused with Tris-EDTA are moistened with $20 \mu \mathrm{l}$ of sterile saline and inoculated with several colonies of the test organisms are aseptically placed onto the MHA next to the cefoxitin disc (almost touching) [45, 68]. Reporting of results is done after 16-20 hours of incubation in ambient air at $35^{\circ} \mathrm{C} \pm 2^{\circ} \mathrm{C}$ as follows; flattening or indentation of the cefoxitin inhibition zone in the vicinity of the test disc is indicative of AmpC production whereas absence of flattening or indentation of the cefoxitin inhibition zone in the vicinity of the test disc is indicative of $\mathrm{AmpC}$ non production $[45,68]$.

The disc approximation method is performed using E.coli ATCC 25922 and inducing substrates; imipenem (10 $\mu \mathrm{g})$, cefoxitin $(30 \mu \mathrm{g})$ and amoxicillin clavulanic acid $(20 / 10 \mu \mathrm{g})$ discs $[64,68,78,80,81]$ as follows, a $0.5 \mathrm{McF}$ arland bacterial suspension of E.coli ATCC 25922 is inoculated onto a plate of MHA. Then onto the MHA plate is placed a ceftazidime $(30 \mu \mathrm{g})$ or cefotaxime $(30 \mu \mathrm{g})$ or phenyl-boronic acid $(400 \mu \mathrm{g})$ (in the center) and at a distance of $20 \mathrm{~mm}$ from this disc, discs of imipenem $(10 \mu \mathrm{g})$, amoxicillin/clavulanic acid $(20 / 10 \mu g)$. Reporting of results is done after 16-20 hours of incubation in ambient air at $35^{\circ} \mathrm{C} \pm 2^{\circ} \mathrm{C}$ as follows; any obvious blunting or flattening of the zone of inhibition between the ceftazidime or cefotaxime or phenyl-boronic acid disc and the inducing substrates 
(imipenem , cefoxitin and amoxicillin/clavulanic acid) is indicative of AmpC production [64, $68,78,80,81]$.

In the three dimensional extract test, $50 \mathrm{mls}$ of a bacterial suspension adjusted to a 0.5 McFarland turbidity standard prepared from an overnight culture on blood agar is inoculated into $12 \mathrm{mls}$ of tryptic soy broth [77, 80, 82, 83, 85]. Following this, the culture is grown at $35^{\circ} \mathrm{C}-37^{\circ} \mathrm{C}$ for $4 \mathrm{hrs}[77,80,82,83,85]$. The bacterial cells are centrifuged and the crude enzyme prepared by freezing-thawing the bacterial cell pellets five times [77, $80,82,83,85]$. The surface of the MHA plate to be used is then inoculated with E.coli ATCC 25922 or E.coli ATCC 11775 following the CLSI guidelines for the standard diffusion method $[77,80,82,83,85]$. A cefoxitin $(30 \mu \mathrm{g})$ disc is then aseptically placed on the inoculated MHA plate $[77,80,82,83,85]$. A linear slit $(3 \mathrm{~mm})$ beginning $3 \mathrm{~mm}$ from the edge of the disc is then cut in the agar in an outward direction using a sterile scalpel blade and a small circular well is made at the other end of the slit near the disc $[80,82,83]$. Then, 30-40 $\mu \mathrm{l}$ of enzyme preparation is dispensed into the small circular well using a pipette at a $10 \mu \mathrm{l}$ increments, beginning near the disc and moving outwards with care being taken not to over fill the slits $[77,80,82,83,85]$. The inoculated media is then incubated at $35^{\circ} \mathrm{C}-37^{\circ} \mathrm{C}$ for $18-24 \mathrm{hrs}$ after allowing the liquid to dry in an upright position $[77,80,82,83,85]$. Interpretation of the results is done following incubation as follows;

three results may be reported, these are isolates showing clear distortion in the zone of inhibition of cefoxitin are reported as AmpC producers, isolates with no clear distortion as AmpC non producers and isolates with minimal distortion as intermediate strains $[77,82$, 83, 85].

\section{Phenotypic Detection of Carbapenemase Production}

Phenotypic detection of carbapenemase production in bacteria is achieved by performing either; i) the modified Hodge test (MHT) [38, 84, 86-91] or ii) the modified carbapenem inactivation method ( $\mathrm{mCIM}$ ), a modification of the carbapenem inactivation method (CIM) [92-94]. These methods have not only been documented to be simple and cost effective but have also been documented to have high sensitivity [38, 84, 86-90]. Despite the high sensitivity of MHT, it has been linked to a high frequency of false-positive results 
especially in carbapenem resistant Enterobacteriaceae that are producers of ESBLs and AmpC $\beta$-lactamases and low sensitivity in regards the detection of NDM-1 producing bacteria [95, 96]. In addition to this, the use of E.coli ATCC 25922 has also been implicated with low sensitivity, specificity and repeatability of MHT [91, 96]. However, replacing E.coli ATCC 25922 with Klebsiella pneumoniae ATCC 700603 has been shown to provide high sensitivity, specificity and repeatability of the test [91]. MHT has also been associated with inability to discriminate between the different classes of carbapenemases (i.e. Klebsiella pneumoniae carbapenemase, Metallo $\beta$-lactamase and Oxacillinases) and also difficulty in interpretation of results has been reported [97]. Despite these, MHT remains a phenotypic reference method for confirmation of carbapenemase production [97].

In MHT, a 0.5 McFarland dilution of Klebsiella pneumoniae ATCC 700603, an indicator strain in $5 \mathrm{ml}$ of sterile saline or nutrient broth is prepared [38, 86-90]. Following this, a $1: 10$ dilution of the indicator strain is streaked as a lawn onto an MHA plate using a sterile swab [38, 86-90]. Then, a meropenem $(10 \mu \mathrm{g})$ or ertapenem $(10 \mu \mathrm{g})$ or imipenem $(10 \mu \mathrm{g})$ disc is placed at the center of the test area on the MHA plate [38, 86-90, 98]. The test organism(s), positive control and negative control are then streaked in straight lines from the edge of the disc to the edge of the plate [38, 86-90]. Klebsiella pneumoniae ATCC BAA-1705 and Klebsiella pneumoniae ATCC-1706 are used as positive and negative controls respectively, these are ran with each batch of the test [38, 86-90]. Reporting of results is done after $16-20$ hours of incubation in ambient air at $35^{\circ} \mathrm{C} \pm 2^{\circ} \mathrm{C}$ as follows; the test organism(s) is positive for MHT when a clover leaf-like indentation of the Klebsiella pneumoniae ATCC 700603 growing along the test organism growth streak within the disc diffusion zone is observed [38, 86-90]. MHT negative organism(s) show no growth of the Klebsiella pneumoniae ATCC 700603 along the test organism growth streak within the disk diffusion [38, 86-90].

In the mCIM method, a sterile inoculating loop is used to add $1 \mu \mathrm{l}$ of the test organism to a tube containing $2 \mathrm{mls}$ of Tryptic soy broth [92, 93]. Then the bacterial suspension is mixed by vortexing for 15 seconds [92, 93]. This is then followed by aseptically adding a commercially available meropenem disk $(10 \mu \mathrm{g})$ to the bacterial suspension [92, 93]. 
Incubation of the bacterial suspension containing the Meropenem disk is then done at $35^{\circ} \mathrm{C}-37^{\circ} \mathrm{C}$ in ambient air for $4 \mathrm{hrs} \pm 15$ minutes [92, 93]. Before the completion of the incubation time, a suspension of the $\mathrm{mCIM}$ indicator organism, a carbapenem susceptible Klebsiella pneumoniae ATCC 25922 with a turbidity equivalent to a 0.5 McFarland is prepared and the surface of an MHA plate is inoculated following the standard disk diffusion method as recommended by the CLSI and placing onto the agar plate the meropenem disc removed aseptically from the Tryptic soy Broth suspension after dragging it on the walls of the tube to drain off the excess fluid [92, 93]. Incubation of the plate is then done at $35^{\circ} \mathrm{C} \pm 2^{\circ} \mathrm{C}$ in ambient air for 18-24hrs [92, 93]. The test is interpreted after measuring the inhibition zone diameter of the meropenem disk as follows; inhibition zone diameters of 6-10 $\mathrm{mm}$ are reported as positive for carbapenemase production, 11$19 \mathrm{~mm}$ as intermediate results and $\geq 20 \mathrm{~mm}$ as negative for carbapenemase production [92, 93]. Another interpretation criteria has been previously described, in this criteria, uninhibited growth of the indicator strain is indicative of carbapenemase production whereas inhibition zone diameters of $\geq 20 \mathrm{~mm}$ are indicative of non-carbapenemase production [92, 93, 99].

To differentiate between the different classes of carbapenemases, novel boronic acid and EDTA based methods (double-disk synergy tests and combined-disk tests) using imipenem, meropenem and ertapenem, in combination with 3-aminophenylboronic acid and ethylene diamine tetra acetic acid have been documented as confirmatory tests [100102].

Detection of Metallo $\beta$-lactamase production in bacteria can be achieved by using two methods, namely; the imipenem-ethylene diamine tetra acetic acid method and another double disc synergy method that involves the use of a ceftazidime $(30 \mu \mathrm{g})$ disc and a

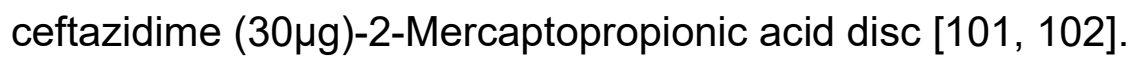

In the imipenem-EDTA method, testing is performed by inoculating the test organism(s) onto MHA for the standard diffusion method as recommended by the CLSI [101]. This is then followed by adding to the MHA plates two commercially available imipenem (10 $\mu \mathrm{g})$ discs, one of the discs un-supplemented with EDTA and another supplemented with 
EDTA at a distance of $15 \mathrm{~mm}$ center to center; However narrower distances have been associated with high test sensitivity and have been previously described [101-103]. The supplementation of the imipenem disc is done by adding $10 \mu \mathrm{l}$ of $0.5 \mathrm{M}$ EDTA to obtain the desired concentration of $1000 \mu \mathrm{g}[87,89,101]$. A concentration of $750 \mu \mathrm{g}$ can also be used in the testing [101]. The 0.5M EDTA stock solution is prepared by dissolving $186.1 \mathrm{~g}$ of disodium EDTA. $2 \mathrm{H}_{2} \mathrm{O}$ in $1000 \mathrm{ml}$ of distilled water and adjusting the solution $\mathrm{PH}$ to 8.0 using $\mathrm{NaOH}$ [101]. After preparation of the mixture, it is sterilized by autoclaving [101]. Incubation of the MHA plates is done at $35^{\circ} \mathrm{C} \pm 2^{\circ} \mathrm{C}$ for $18-24 \mathrm{hrs}$ in ambient air [87, 89, 101]. These tests are interpreted after measuring the inhibition zone diameters around the imipenem disc not supplemented with EDTA and the imipenem discs supplemented with EDTA as follows; the inhibition zone diameters of the imipenem disc supplemented with EDTA are each compared to the inhibition zone diameter of the un-supplemented imipenem disc and an increase in inhibition zone diameter of $\geq 5 \mathrm{~mm}$ in the EDTAsupplemented disc is interpreted as positive for Metallo $\beta$-lactamase production [87, 89, 101]. Noteworthy, the Supplemented discs prepared maybe kept at $4^{\circ} \mathrm{C}$ or at $-20^{\circ} \mathrm{C}$ in airtight vials without desiccant and under these conditions, these remain stable for 12 and 16 weeks respectively [101].

In the ceftazidime disc method, a ceftazidime disc and another supplemented with 2Mercaptopropionic acid are used [101]. In this method, the procedure and interpretation is done as documented in the previous method, the imipenem-EDTA [101].

The detection and differentiation of Klebsiella pneumoniae carbapenemase production and Metallo $\beta$-lactamase production can be achieved using a phenotypic algorithm that involves the use of three combined-disc tests [16, 104-107]. These tests consist of i) Ertapenem alone and ertapenem supplemented with phenylboronic acid (PBA); ii) ertapenem alone and ertapenem supplemented with ethylene diamine tetra acetic acid (EDTA); iii) both tests [16, 104-107]. Additionally, these tests can be used to detect coproduction of both carbapenemases in bacteria [16, 107]. In these tests, the concentrations of the PBA and EDTA used are $400 \mu \mathrm{g}$ of PBA and $292 \mu \mathrm{g}$ of EDTA [16, 106]. The stock solution of PBA is prepared by dissolving PBA in DMSO at a 
concentration of $20 \mathrm{mg} / \mathrm{mL}[16,106,108]$. From this solution, $20 \mu \mathrm{l}$ (Containing $400 \mu \mathrm{g}$ of PBA) are then added onto commercially available meropenem discs to obtain the PBA supplemented meropenem discs [16, 106, 108]. Similarly, the stock solution of EDTA is prepared by dissolving anhydrous EDTA (Sigma-Aldrich) in distilled water at a concentration of $0.1 \mathrm{M}[16,106,108]$. From this solution, $10 \mu \mathrm{l}$ (containing $292 \mu \mathrm{g}$ of EDTA) are then added onto commercially available meropenem discs to obtain the EDTA supplemented meropenem discs [16, 106, 108]. Following disc preparation, the discs are dried and used within 60 minutes [16]. Testing is performed by inoculating MHA for the standard diffusion method as recommended by the CLSI and placing onto agar plates a disc of meropenem that is not supplemented with any of the inhibitors, (PBA and EDTA) and three discs of meropenem supplemented with $400 \mu \mathrm{g}$ of PBA, $292 \mu \mathrm{g}$ of EDTA or both $400 \mu \mathrm{g}$ of PBA and $292 \mu \mathrm{g}$ of EDTA [16, 106]. Incubation of the agar plates is done at $35^{\circ} \mathrm{C} \pm 2^{\circ} \mathrm{C}$ for $18-24 \mathrm{hrs}[16,106,107]$. These tests are interpreted after measuring the inhibition zone diameters around the meropenem disc not supplemented with any inhibitor and the meropenem discs supplemented with either PBA, EDTA or both PBA and EDTA as follows; the inhibition zone diameters of the meropenem disc supplemented with either PBA, EDTA or both PBA and EDTA are each compared to the inhibition zone diameter of the un-supplemented meropenem disc $[16,106]$. An increase in the inhibition zone diameter in any of the supplemented meropenem discs by $\geq 5 \mathrm{~mm}$ is considered positive for Klebsiella pneumoniae carbapenemase production [16, 106]. Bacterial isolates are considered positive for KPC and MBL co-production only when, the inhibition zone diameter around the meropenem disc supplemented with both PBA and EDTA is $\geq 5 \mathrm{~mm}$ compared to the inhibition zone diameter of the meropenem disc not supplemented with any inhibitor while the inhibition zone diameters of the meropenem disc supplemented with PBA and that supplemented with EDTA each are $\leq 5 \mathrm{~mm}$ compared to the meropenem disc not supplemented with any of the inhibitors $[16,106]$.

Boronic acid based tests can also be used to detect ESBL production in KPC positive isolates [106, 108]. In this testing, combined disc tests are used, namely; i) cefotaxime (CTX) with or without clavulanic acid (CA) supplemented with boronic acid (BA) and ii) ceftazidime (CAZ) with or without clavulanic acid supplemented with boronic acid [106, 
108]. The stock solution of BA is prepared by dissolving phenylboronic acid (PBA) in dimethyl sulfoxide and water at a concentration of $20 \mathrm{mg} / \mathrm{ml}$. From the stock solution, 20 $\mu \mathrm{l}$ are added onto commercially available discs containing CTX $(30 \mu \mathrm{g})$ or CAZ $(30 \mu \mathrm{g})$ with or without CA $(10 \mu \mathrm{g})$. Addition of this volume to either of the discs makes the final volume on the discs $400 \mu \mathrm{g}[106,108]$. Following this, discs are dried and used within 60 minutes [106]. Testing is performed by inoculating MHA for the standard diffusion method as recommended by the CLSI and placing onto agar plates a disC of CAZ or CTX with or without CA that is not supplemented with BA and two discs of CAZ or CTX with or without CA supplemented with $400 \mu \mathrm{g}$ of BA $[16,106]$. These tests are interpreted after measuring the inhibition zone diameters around the CAZ or CTX disc with or without CA not supplemented with BA and the CTX or CAZ disc with or without CA supplemented with $B A$ as follows; the inhibition zone diameters of the CTX or CAZ disc with or without CA disc supplemented with $B A$ are each compared to the inhibition zone diameter of the un-supplemented CTX or CAZ disc with or without CA [106]. An increase in the inhibition zone diameter in any of the supplemented CAZ or CTX discs with or without CA by $\geq 5$ $\mathrm{mm}$ is considered positive for ESBL production [106].

\section{Conclusion}

Different phenotypic screening and confirmation methods have overtime been developed and evaluated for the detection of extended spectrum $\beta$-lactamases and carbapenemases in clinically significant gram negative bacteria. In this review we summarize approved guidelines, the different methods described in literature for the detection of these enzymes and also methods that discourse challenges mostly encountered during detection of these enzymes in clinical microbiology laboratories. In this section, we provide recommendations on the best and/or acceptable methods that might be useful in formulating efficient approaches for optimizing the detection of extended spectrum $\beta$-lactamases and carbapenemases in clinically significant bacteria in microbiology laboratories.

It is recommended that screening for ESBL production in bacteria be done using the cephalosporin/clavulanate combination disc diffusion and broth micro-dilution methods using cefotaxime, ceftazidime and cefpodoxime (for testing using a single drug). Testing 
with more than one antibiotic is also recommended as it increases sensitivity of the testing. The modified double disc synergy test using amoxicillin-clavulanic acid (20/10 $\mu \mathrm{g})$ or piperacillin tazobactam $(100 / 10 \mu g)$ along two third generation cephalosporins, ceftazidime $(30 \mu \mathrm{g})$ and cefotaxime $(30 \mu \mathrm{g})$; a fourth generation cephalosporin, cefepime $(30 \mu \mathrm{g})$ used as a replacement for ceftriaxone $(30 \mu \mathrm{g})$ and aztreonam $(30 \mu \mathrm{g})$ remains the preferred method for confirmation of ESBL production, this not only confirms ESBL production but also ably distinguishes ESBL production and over-expression of AmpC derepressed mutants. Additionally, the method also sets precedence for AmpC screening in bacteria.

In the detection of AmpC production, screening of isolates may be done using the MDDS and the cefoxitin/AmpC inhibitor combination disc method, using cloxacillin and phenylboronic acid, confirmation of AmpC production can then be done by using either the AmpC disc test, the disc approximation test or the modified three dimensional extract test.

It is also recommended that confirmation of carbapenemase production be done with the modified hodge test, using Klebsiella pneumoniae ATCC 700603 as the indicator organism or the modified carbapenem inactivation method. However, to differentiate between the different classes of carbapenemases, boronic acid and EDTA based methods (double-disk synergy tests and combined-disk tests) using imipenem, meropenem and ertapenem, in combination with 3-aminophenylboronic acid and ethylene diamine tetra acetic acid can be used.

Detection of Metallo $\beta$-lactamase production in bacteria can be achieved by using two methods, namely; the imipenem-ethylene diamine tetra acetic acid method and another double disc synergy method that involves the use of a ceftazidime $(30 \mu \mathrm{g})$ disc and a ceftazidime (30 $\mu$ g)-2-Mercaptopropionic acid disc.

Detection and differentiation of Klebsiella pneumoniae carbapenemase production and Metallo $\beta$-lactamase production can be achieved using a phenotypic algorithm that involves the use of three combined-disc tests, these tests consist of i) Ertapenem alone and ertapenem supplemented with phenylboronic acid (PBA); ii) Ertapenem alone and ertapenem supplemented with ethylene diamine tetra acetic acid (EDTA); iii) both tests. 


\section{Declarations}

\subsection{Ethical approval and consent to participate}

Not applicable

\subsection{Consent for publication}

Not applicable

\subsection{Conflicting interests}

Not applicable

\subsection{Author contributions}

DA, Drafted, Edited and Reviewed Original Manuscript, Drafted and Reviewed Final Manuscript

\subsection{Acknowledgements}

Not applicable

References

1. Rupinder, B., Geeta, W, Shikha, J. Prevalence of extended spectrum b lactamases in multidrug resistant strains of gram negative Bacilli. J. Acad. Indus. Res, 2013. 1(9): p. 558-560.

2. Abhijit, A., Sunita, N, Maria, K. Study of urinary isolates withreferencetoextendedspectrumbetalactamasesdetectionand antibiogram. J. Evol. Med. Dent. Sci, 2013. 2 (9): p. 1049-1055.

3. Majda, Q., Najma, A, Summyia, B. Evaluation of extended spectrum betalactamase mediated resistance in Escherichia coli and Klebsiella in urinary tract infection at a tertiary care hospital. Biomedica 2013 29(78-81).

4. Meeta, S., Sati, P, Preeti, S. Prevalence and antibiogram of extended spectrum blactamase (ESBL) producing Gram negative bacilli and further molecular characterization of ESBL producing Escherichia coli and Klebsiella spp. J.Clin.Diag.Res, 2013 7(10): p. 2168- 2172. 
5. Sidra Shan, S.S., Khawaja Ahmad. Detection of blalMP Gene in Metallo- $\beta$ Lactamase Producing Isolates of Imipenem Resistant Pseudomonas Aeruginosa; an Alarming Threat. Journal of Microbiology Research 2015.5(6): p. 175-80.

6. Sibhghatulla Shaikh, J.F., Shazi Shakil, Syed Mohd, Danish Rizvi, Mohammad Amjad Kamal. Antibiotic resistance and extended spectrum beta-lactamases: Types, epidemiology and treatment. Saudi Journal of Biological Sciences, 2015. 22: p. 90-101.

7. Drieux, L., F. Brossier, W. Sougakoff, and V. Jarlier. Phenotypic detection of extended-spectrum B-lactamase production in Enterobacteriaceae: review and bench guide. Clin. Microbiol. Infect. , 2008. 14(Suppl. 1): p. 90-103.

8. Nordmann, P., et al., Identification and screening of carbapenemase-producing Enterobacteriaceae. Clinical Microbiology and Infection, 2012. 18(5): p. 432-438.

9. Pitout, J.D. and K.B. Laupland. Extended-spectrum $\beta$-lactamase-producing Enterobacteriaceae: an emerging public-health concern. The Lancet infectious diseases, 2008. 8(3): p. 159-166.

10. Pawel Sacha, P.W., Tomasz Hauschild, Marcin Zórawski, Dorota Olszañska, Elzbieta Tryniszewska. Metallo- $\beta$-lactamases of Pseudomonas aeruginosa -a novel mechanism resistance to $\beta$-lactam antibiotics. Folia Histochem Cytobiol. , 2008. 2008:46(2): p. 137 (137-142).

11. Khan, M., S. Thukral, and R. Gaind. Evaluation of a modified double-disc synergy test for detection of extended spectrum $\beta$-lactamases in AMPC $\beta$-lactamaseproducing proteus mirabilis. Indian journal of medical microbiology, 2008. 26(1): p. 58.

12. Bush, K., Jacoby, G. A, Medeiros, A. A. A functional classification scheme for betalactamases and its correlation with molecular structure. Antimicrob. Agents Chemother 1995 39: p. 1211- 1233.

13. Ambler, R.P. The structure of b-lactamases. Philos.Trans.R. Soc. Lond. B. Biol. Sci. , 1980. 289 p. 321-331.

14. Étienne Ruppé, P.L.W.a.F.B. Mechanisms of antimicrobial resistance in Gramnegative bacilli. 2015. 5(21).

15. Steward, C.D., Wallace,D, Hubert,S.K, Lawton,R, Fridkin,S.K, Gaynes, R.P, McGowan, J.E, Tenover, F.C. Ability of laboratories to detect emerging antimicrobial resistance in nosocomial pathogens: a survey of project ICARE laboratories. Diag. Microbiol. Infect. Dis, 2000.38 (1): p. 59-67.

16. Athanassios Tsakris, A.P., Spyros Pournaras, Evangelia Voulgari, Georgia Vrioni , Katerina Themeli-Digalaki, Dimitra Petropoulou and Danai Sofianou. A simple phenotypic method for the differentiation of metallo-b-lactamases and class A KPC 
carbapenemases in Enterobacteriaceae clinical isolates. J Antimicrob Chemother 2010. 65. p. 1664-1671.

17. Pfaller MA, S.J., Overview of the epidemiological profle and laboratory detection of extended-spectrum beta-lactamases. Clin Infect Dis. , 2006 4: p. S153-63.

18. Walsh, C., Where will new antibiotics come from?',.. Nat Rev Microbiol, , (2003). vol. 1, (no.1,): p. pp. 65-70.

19. Paterson, D.L., Ko, W.C,Von, G.A, Casellas,J.M, Mulazimoglu, L, Klugman,K.P, Bonomo,R.A, Rice,L.B, McCormack,J.G, Yu, V.L. Outcome of cephalosporin treatment for serious infections due to apparently susceptible organisms producing extended-spectrum b-lactamases: implications for the clinical microbiology laboratory. J. Clin. Microbiol 2001. 39: p. 2206-2212.

20. Bradford, P.A., Yang, Y, Sahm, D, Grope, I, Gardovska, D, Storch, G. CTX-M-5, a novel cefotaxime-hydrolyzing blactamase from an outbreak of Salmonella typhimurium in Latvia. Antimicrob.Agents Chemother, 1998. 42: p. 1890-1894.

21. Tzouvelekis, L.S., Tzelepi, E, Tassios, P.T, Legakis, N.J. CTX-M type betalactamases: an emerging group of extendedspectrum enzymes. Int. J. Antimicrob. Agents 2000. 14: p. 137-143.

22. Ma, L., Ishii, $\mathrm{Y}$, Ishiguro,M, Matsuzawa, $\mathrm{H}$, Yamaguchi,K. Cloning and sequencing of the gene encoding Toho-2, a class A b-lactamase preferentially inhibited by tazobactam. Antimicrob. Agents Chemother, 1998. 42: p. 1181-1186.

23. Bonnet, R., Growing group of extended-spectrum beta-lactamases: the CTX-M enzymes. . Antimicrob. Agents Chemother, 2004. 48(1): p. 1-14.

24. Sirot, D., Sirot,J, Labia,R, Morand,A, Courvalin,P, Darfeuille Michaud,A , Perroux, R, Cluzel,R. Transferable resistance to third-generation cephalosporins in clinical isolates of Klebsiella pneumoniae: identification of CTX-1, a novel beta lactamase. J. Antimicrob.Chemother, 1987. 20(3): p. 323-334.

25. Soughakoff, W., Goussard, S, Courvalin, P. TEM-3 betalactamases which hydrolyzes broad-spectrum cephalosporins is derived from the TEM-2 penicillinases by two amino acid substitutions. FEMS Microbiol. Lett. , 1988. 56: p. 343-348.

26. Livermore, D.M. Beta-lactamases in laboratory and clinical resistance. Clin. Microbiol, 1995. Rev. 8: p. 557-584.

27. Weldhagen, G.F., Poirel, L, Nordmann, P. Ambler class A extended-spectrum beta-lactamases in Pseudomonas aeruginosa: novel developments and clinical impact. Antimicrob. Agents Chemother, 2003. 47: p. 2385-2392. 
28. Neuhauser, M.M., Weinstein, R.A, Rydman, R, Danziger, L.H, Karam, G, Quinn, J.P. Antibiotic resistance among gramnegative bacilli in US intensive care units: implications for fluoroquinolone use. JAMA 2003. 289: p. 885-888.

29. Naas, T., Poirel, L, Nordmann,P. Minor extended-spectrum b-lactamases. Clin. Microbiol. Infect 2008 14( 1 ): p. 42-52.

30. Bradford, P.A. Extended spectrum beta-lactamases in the 21st century: characterization, epidemiology and detection of this important resistance threat. Clin. Microbiol, 2001. Rev. 14: p. 933-951.

31. Bush, A.M.Q.a.K., Carbapenemases: the Versatile beta-Lactamases. Clinical microbiology reviews, 2007. Vol. 20(No. 3): p. p. 440-458.

32. Nuno T. Antunes, T.L.L., Marta Toth, Nichole K. Stewart, Hilary Frase, Sergei B. Vakulenko. Class D B-Lactamases: Are They All Carbapenemases?. Antimicrobial Agents and Chemotherapy 2014 Volume 58 (Number 4): p. p. 2119-2125.

33. Walsh, T.R., Toleman, M.A, Poirel, L \& Nordmann, P. Metallo-beta-lactamases: the quiet before the storm? . ClinMicrobiolRev, 2005. 18: p. 306-325.

34. Rasmussen BA, B.K. Carbapenem-hydrolyzing beta-lactamases. Antimicrob Agents Chemother, 1997. 41: p. 223-232.

35. Diene S.M, R.J.M. Carbapenemase genes and genetic platforms in gram negative bacilli: Enterobacteriaceae, Pseudomonas and Acinetobacter species. Clin Microbiol Infect, 2014. 20(9).

36. Ambler, R.P., A. F. W. Coulson, J. M. Frere, J. M. Ghuysen, B. Joris, M. Forsman, R. C. Levesque, G. Tiraby, and S. G. Waley. A standard numbering scheme for the class A \& B-lactamases. Biochem. J, 1991. 276: p. 269-270.

37. Walsh, T.R. The emergence and implications of metallo-bata-lactamases in Gramnegative bacteria. . Clin. Microbiol. Infect. , 2005. 11: p. 2-9.

38. Miriagou V, C.G., Edelstein M, Galani I, Giske CG, Gniadkowski M, Malamou-Lada E, Martinez-Martinez L, Navarro F, Nordmann P et al., Acquired carbapenemases in Gram-negative bacterial pathogens: detection and surveillance issues. Clin Microbiol Infect. 2010. 16(2): p. 112-122.

39. Lauretti L, R.M., Mazzariol A. Cloning and Molecular Characterisation of blaVIM, a new integron bourne metallo- beta-lactamase gene from Pseudomonas aeruginosa clinical isolate. Antimicrob Agents Chemother, 1999. 43: p. 1584-1590.

40. Matthew, M., Plasmid mediated B-lactamases of gram-negative bacteria: properties and distribution. J. Antimicrob. Chemother 1979 5: p. 349-358. 
41. Medeiros, A.A. B-Lactamases. Br. Med. Bull, 1984. 40: p. 18-27.

42. Simpson, I.N., P. B. Harper, and C. H. O'Callaghan. Principal B-lactamases responsible for resistance to B-lactam antibiotics in urinary tract infections. Antimicrob. Agents Chemother 1980. 17: p. 929-936.

43. Bush, K., Recent developments in 8-lactamase research and their implications for the future Rev. Infect. Dis, 1988. 10: p. 681-690; 739-743.

44. Oberoi, L., et al. ESBL, MBL and Ampc $\beta$ lactamases producing superbugs-Havoc in the Intensive Care Units of Punjab India. Journal of clinical and diagnostic research: JCDR, 2013. 7(1): p. 70.

45. Singhal, S., et al. Evaluation of methods for AmpC beta-lactamase in gram negative clinical isolates from tertiary care hospitals. Indian journal of medical microbiology, 2005. 23(2): p. 120.

46. Shoorashetty, R., T. Nagarathnamma, and J. Prathibha. Comparison of the boronic acid disk potentiation test and cefepime-clavulanic acid method for the detection of ESBL among AmpC-producing Enterobacteriaceae. Indian journal of medical microbiology, 2011. 29(3): p. 297.

47. Hageman, J.C., et al., Antimicrobial proficiency testing of National Nosocomial Infections Surveillance System hospital laboratories. Infection Control \& Hospital Epidemiology, 2003. 24(5): p. 356-361.

48. Tenover, F.C., et al., Ability of laboratories to detect emerging antimicrobial resistance: proficiency testing and quality control results from the World Health Organization's external quality assurance system for antimicrobial susceptibility testing. Journal of clinical microbiology, 2001. 39(1): p. 241-250.

49. Pitout, J.D., et al., Emergence of Enterobacteriaceae producing extendedspectrum $\beta$-lactamases (ESBLs) in the community. Journal of Antimicrobial Chemotherapy, 2005. 56(1): p. 52-59.

50. Stevenson, K.B., et al., Detection of antimicrobial resistance by small rural hospital microbiology laboratories: comparison of survey responses with current NCCLS laboratory standards. Diagnostic microbiology and infectious disease, 2003. 47(1): p. 303-311.

51. Col, N.G., A.S. Brig, and S.B. RetdCol, Therapeutic challenges of ESBLS and $A m p C$ beta-lactamase producers in a tertiary care center. Medical Journal Armed Forces India, 2013. 69(1): p. 4-10.

52. Queenan, A.M., et al., Effects of inoculum and $\beta$-lactamase activity in AmpC-and extended-spectrum $\beta$-lactamase (ESBL)-producing Escherichia coli and Klebsiella 
pneumoniae clinical isolates tested by using NCCLS ESBL methodology. Journal of clinical microbiology, 2004. 42(1): p. 269-275.

53. Willems, E., et al., Towards a phenotypic screening strategy for emerging $\beta$ lactamases in Gram-negative bacilli. International journal of antimicrobial agents, 2013. 41(2): p. 99-109.

54. Legrand P, F.G., Bure A et al. , Detection of extended broad-spectrum betalactamases in Enterobacteriaceae in four French hospitals. Eur J Clin Microbiol Infect Dis 1989. 8. p. 527-529.

55. M'Zali, F.H., et al., Detection of extended-spectrum $\beta$-lactamases in members of the family Enterobacteriaceae: comparison of the MAST DD test, the double disc and the Etest ESBL. Journal of Antimicrobial Chemotherapy, 2000. 45(6): p. 881885.

56. Thomson KS, S.C., Detection of extendedspectrum beta-lactamases in members of the family Enterobacteriaceae: comparison of the double-disk and threedimensional tests. Antimicrob Agents Chemother.1992. 36. p. 1877-1882.

57. Rawat, D. and D. Nair, Extended-spectrum $\beta$-lactamases in Gram Negative Bacteria. Journal of global infectious diseases, 2010. 2(3): p. 263.

58. (CLSI), C.a.L.S.I., Performance Standards for Antimicrobial Susceptibility Testing. CLSI supplement M100. Clinical and Laboratory Standards, 2018. 28th ed.

59. Livermore, D.M. and D.L. Paterson, Pocket Guide to Extended-spectrum [beta]lactamases in Resistance. 2006: Current Medicine Group.

60. Steward, C.D., et al., Characterization of clinical isolates of Klebsiella pneumoniae from 19 laboratories using the National Committee for Clinical Laboratory Standards extended-spectrum $\beta$-lactamase detection methods. Journal of clinical microbiology, 2001. 39(8): p. 2864-2872.

61. Jarlier, V., et al., Extended broad-spectrum $\beta$-lactamases conferring transferable resistance to newer $\beta$-lactam agents in Enterobacteriaceae: hospital prevalence and susceptibility patterns. Clinical Infectious Diseases, 1988. 10(4): p. 867-878.

62. Drieux, L., et al., Phenotypic detection of extended-spectrum $\beta$-lactamase production in Enterobacteriaceae: review and bench guide. Clinical Microbiology and Infection, 2008. 14: p. 90-103.

63. Kaur, J., S. Chopra, and G.M. Sheevani. Modified double disc synergy test to detect ESBL production in urinary isolates of Escherichia coli and Klebsiella pneumoniae. Journal of clinical and diagnostic research: JCDR, 2013. 7(2): p. 229. 
64. Garrec, H., et al., Comparison of Nine Phenotypic Methods for Detection of Extended-Spectrum ß-Lactamases (ESBL) Production by Enterobacteriaceae. Journal of clinical microbiology, 2011.

65. JaSpal Kaur, S.C., Sheevani, GoMty MahaJan. Modifed Double Disc Synergy Test to Detect ESBL Production in Urinary Isolates of Escherichia coli and Klebsiella pneumoniae. Journal of Clinical and Diagnostic Research. , 2013 Vol-7(2): p. 229233.

66. Jarlier V, N.M., Fournier G, Philippon A. Extended broad-spectrum betalactamases conferring transferable resistance to newer beta-lactam agents in Enterobacteriaceae: hospital prevalence and susceptibility patterns. Rev Infect Dis 1988. 10: p. 867-878.

67. Tzelepi E, G.P., Sofianou D, Loukova V, Kemeroglou A, Tsakris A. Detection of extended-spectrum beta-lactamases in clinical isolates of Enterobacter cloacae and Enterobacter aerogenes. J Clin Microbiol 2000. 38: p. 542-546.

68. Soha A. El-Hady, L.A.A., Occurrence and detection of AmpC b-lactamases among Enterobacteriaceae isolates from patients at Ain Shams University Hospital. The Egyptian Journal of Medical Human Genetics 2015. 16: p. 239-244.

69. Jacoby, G.A., AmpC $\beta$-lactamases. Clinical microbiology reviews, 2009. 22(1): p. 161-182.

70. Hanson, N. and C. Sanders. Regulation of inducible AmpC beta-lactamase expression among Enterobacteriaceae. Current pharmaceutical design, 1999. 5(11): p. 881-894.

71. Thomson, K.S. Controversies about Extended-Spectrum and AmpC BetaLactamases. Emerging Infectious Diseases, 2001. Vol. 7 (No. 2).

72. Tan, T.Y., Ng, L. S, He, J, Koh, T. H. \& Hsu, L. Y. Evaluation of screening methods to detect plasmid-mediated AmpC in Escherichia coli, Klebsiella pneumoniae, and Proteus mirabilis. Antimicrob Agents Chemother 2009. 53: p. 146-149.

73. Jones, R.N., et al., Inducible $\beta$-lactamase-mediated resistance to third-generation cephalosporins. Clinical Microbiology and Infection, 1997. 3: p. s7-s20.

74. Aggeliki Poulou, E.G., Georgia Vrioni, Vassiliki Koumaki, Theodoros Pittaras, Spyros Pournaras, Athanassios Tsakrisa. Modified CLSI Extended-Spectrum 6Lactamase (ESBL) Confirmatory Test for Phenotypic Detection of ESBLs among Enterobacteriaceae Producing Various beta-Lactamases. Journal of Clinical Microbiology, 201452 (5): p. p. 1483-1489.

75. Brenwald, N.P., Jevons, G, Andrews, J, Ang, L. \& Fraise, A. P. Disc methods for detecting AmpC b-lactamase-producing clinical isolates of Escherichia coli and Klebsiella pneumoniae. J Antimicrob Chemother 2005. 56: p. 600-601. 
76. Ingram, P.R., et al., Comparison of methods for AmpC $\beta$-lactamase detection in Enterobacteriaceae. Journal of medical microbiology, 2011. 60(6): p. 715-721.

77. M'Zali, F.H., J. Heritage, D. M. Gascoyne-Binzi, M. Denton, N. J. Todd, and P. M. Hawkey, Transcontinental importation into the UK of Escherichia coli expressing a plasmid-mediated AmpC-type beta-lactamase exposed during an outbreak of SHV-5 extended-spectrum beta-lactamase in a Leeds hospital. J. Antimicrob. Chemother, 1997. 40: p. 823-831.

78. S. Peter-Getzlaff, S.P., M. Poledica, M. Hombach, J. Giger, E. C. Böttger , R. Zbinden and G.V. Bloemberg, Detection of AmpC beta-lactamase in Escherichia coli: comparison of 1 three phenotypic confirmation assays and genetic analysis. J. Clin. Microbiol doi:10.1128/JCM.00091-11.

79. Coudron, P.E. Inhibitor-based methods for detection of plasmid-mediated AmpC beta-lactamases in Klebsiella spp., Escherichia coli, and Proteus mirabilis. J Clin Microbiol 2005. 43: p. 4163-4167.

80. Tan, T.Y., et al., Evaluation of screening methods to detect plasmid-mediated AmpC in Escherichia coli, Klebsiella pneumoniae, and Proteus mirabilis. Antimicrobial agents and chemotherapy, 2009. 53(1): p. 146-149.

81. Polsfuss, S., et al., A practical approach for reliable detection of AmpC betalactamase producing Enterobacteriaceae. Journal of clinical microbiology, 2011: p. JCM. 00404-11.

82. Coudron, P.E., E.S. Moland, and K.S. Thomson. Occurrence and detection of AmpC beta-lactamases among Escherichia coli, Klebsiella pneumoniae, and Proteus mirabilis isolates at a veterans medical center. Journal of Clinical Microbiology, 2000. 38(5): p. 1791-1796.

83. Thomson, K.S., and C. C. Sanders, Detection of extended-spectrum b-lactamases in members of the family Enterobacteriaceae: comparison of the double-disk and three-dimensional tests. Antimicrob. Agents Chemother., 1992. 36.p. 1877-1882.

84. Hemalatha, V., et al., Detection of Amp C beta lactamases production in Escherichia coli \& Klebsiella by an inhibitor based method. Indian journal of medical research, 2007. 126(3): p. 220.

85. Vercauteren, E., P. Descheemaeker, M. leven, C. C. Sanders, and H. Goossens. Comparison of screening methods for detection of extendedspectrum $b$ lactamases and their prevalence among blood isolates of Escherichia coli and Klebsiella spp. in a Belgian teaching hospital. J. Clin. Microbiol, 1997. 35: p. 21912197.

86. Amjad A, M.I., Abbasi SA, Farwa U, Malik N, Zia F. Modifed Hodge test: A simple and effective test for detection of carbapenemase production. Iranian Journal of Microbiology, 2011 Volume 3(Number 4): p. 189-193. 
87. Kateete, D.P., Ritah Nakanjako, Juliet Namugenyi, Joseph Erume, Moses L Joloba, and Christine F Najjuka. Carbapenem Resistant Pseudomonas Aeruginosa and Acinetobacter Baumannii at Mulago Hospital in Kampala, Uganda (2007-2009). SpringerPlus 5 (1). Cham: Springer International Publishing: 1308., 2016.

88. Okoche, D., Benon B Asiimwe, Fred Ashaba Katabazi, Laban Kato, and Christine F Najjuka. Prevalence and Characterization of Carbapenem-Resistant Enterobacteriaceae Isolated from Mulago National Referral Hospital, Uganda. PLOS ONE 10 (8). Public Library of Science: e0135745., 2015.

89. Asthana, S., P. Mathur, and V. Tak. Detection of carbapenemase production in Gram-negative bacteria. Journal of laboratory physicians, 2014. 6(2): p. 69.

90. Laboratory Protocol for Detection of Carbapenem-Resistant or CarbapenemaseProducing, K.s.a.E.c.f.R.S., Department of Health and Human Services. Centres for Disease Control and Prevention.

91. Pasteran, F., et al., Sensitive and specific Modified Hodge Test for KPC and metallo-beta-lactamase detection in Pseudomonas aeruginosa by use of a novel indicator strain: Klebsiella pneumoniae ATCC 700603. Journal of clinical microbiology, 2011: p. JCM. 05602-11.

92. Virginia M. Pierce, P.J.S., David R. Lonsway, Darcie E. Roe-Carpenter,d J. Kristie Johnson, William B. Brasso, April M. Bobenchik, Zabrina C. Lockett, Angella, Charnot-Katsikas, Mary Jane Ferraro, Richard B. Thomson, Jr., Stephen G. Jenkins, Brandi M. Limbago, Sanchita Das. The Modified Carbapenem Inactivation Method (mCIM) for Phenotypic Detection of Carbapenemase Production among Enterobacteriaceae. J. Clin. Microbiol., 2017.

93. van der Zwaluw K, d.H.A., Pluister GN, Bootsma HJ, de Neeling AJ, Schouls LM. The carbapenem inactivation method (CIM), a simple and low-cost alternative for the Carba NP test to assess phenotypic carbapenemase activity in gram-negative rods. PLoS One. 2015. 10(e0123690).

94. Pierce, V.M., et al., The modified carbapenem inactivation method (mClM) for phenotypic detection of carbapenemase production among Enterobacteriaceae. Journal of clinical microbiology, 2017: p. JCM. 00193-17.

95. Hrabák, J., E. Chudáčková, and C. Papagiannitsis. Detection of carbapenemases in E nterobacteriaceae: a challenge for diagnostic microbiological laboratories. Clinical Microbiology and Infection, 2014. 20(9): p. 839-853.

96. Pasteran, F., et al., Controlling false-positive results obtained with the Hodge and Masuda assays for detection of class $A$ carbapenemase in species of Enterobacteriaceae by incorporating boronic acid. Journal of clinical microbiology, 2010. 48(4): p. 1323-1332. 
97. Bialvaei, A.Z., et al., Current methods for the identification of carbapenemases. Journal of Chemotherapy, 2016. 28(1): p. 1-19.

98. Deshpande, L.M., R. N. Jones, T. R. Fritsche, and H. S. Sader. Occurrence and characterization of carbapenemase-producing Enterobacteriaceae: report from the SENTRY Antimicrobial Surveillance Program (2000-2004). Microb. Drug Resist, 2006. 12: p. 223-230.

99. Tijet N, P.S., Melano RG. Detection of carbapenemase activity in Enterobacteriaceae: comparison of the carbapenem inactivation method versus the Carba-NP test. J Antimicrob Chemother 2016. 71: p. 274-276.

100. Tsakris, A., et al., Comparative evaluation of boronic acid combined-disk tests for the detection of KPC carbapenemase-producing Enterobacteriaceae clinical isolates. Journal of clinical microbiology, 2011: p. JCM. 00666-11.

101. Dongeun Yong, K.L., Jong Hwa Yum, Hee Bong Shin, Gian Maria Rossolini, and Yunsop Chong. Imipenem-EDTA Disk Method for Differentiation of Metallo-6Lactamase-Producing Clinical Isolates of Pseudomonas spp. and Acinetobacter spp.Journal of clinical microbiology.2002. Vol. 40(No. 10): p. p. 3798-3801

102. Arakawa, Y., N. Shibata, K. Shibayama, H. Kurokawa, T. Yagi, H. Fujiwara, and M. Goto. Convenient test for screening metallo-6-lactamase-producing gramnegative bacteria by using thiol compounds. J. Clin. Microbiol. 2000. 38: p. 40-43.

103. Lee, K., Y. Chong, H. B. Shin, Y. A. Kim, D. Yong, and J. H. Yum. Modified Hodge and EDTA-disk synergy tests to screen metallo-6-lactamase-producing strains of Pseudomonas and Acinetobacter species Clin. Microbiol. Infect. 2001. 7: p. 88-91.

104. Song W, B.I., Lee YN et al., Detection of extended-spectrum b-lactamases by using boronic acid as an AmpC b-lactamase inhibitor in clinical isolates of Klebsiella spp. and Escherichia coli. . J Clin Microbiol. 2007. 45. p. 1180-4.

105. Tsakris A, P.A., Themeli-Digalaki K et al., Use of boronic acid disk tests to detect extended-spectrum b-lactamases in clinical isolates of KPC carbapenemasepossessing Enterobacteriaceae. . J Clin Microbiol. 2009.47: p. 3420-6.

106. Athanassios Tsakris, A.P., Katerina Themeli-Digalaki, Evangelia Voulgari, Theodore Pittaras, Danai Sofianou, Spyros Pournaras, and Dimitra Petropoulou. Use of Boronic Acid Disk Tests To Detect ExtendedSpectrum B-Lactamases in Clinical Isolates of KPC Carbapenemase-Possessing Enterobacteriaceae. Journal of clinical microbiology. 2009. Vol. 47, No. 1: p. p. 3420-3426.

107. Pournaras, S., Aggeliki Poulou, and Athanassios Tsakris. Inhibitor-Based Methods for the Detection of KPC Carbapenemase-Producing Enterobacteriaceae in Clinical Practice by Using Boronic Acid Compounds. Journal of Antimicrobial Chemotherapy 2010. 65 (7): p. 1319-21. 
108. PE, C., Inhibitor-based methods for detection of plasmid-mediated AmpC blactamases in Klebsiella spp., Escherichia coli, and Proteus mirabilis. J Clin Microbiol 2005. 43: p. 4163-7. 